\title{
Human papillomavirus infection of the uterine cervix: histological appearances in 28 cases identified by immunohistochemical techniques
}

\author{
JL DYSON, ${ }^{*}$ PG WALKER, A SINGER
}

From the Departments of *Pathology and Gynaecology, Royal Northern Hospital and Whittington Hospital, London

SUMMARY Twenty eight biopsy specimens of the cervix showed positive immunohistochemical staining when treated with an antiserum raised against an internal capsid antigen of human papillomavirus (HPV). Histological examination of adjoining sections from the same blocks showed a much wider range of abnormalities than those already described in association with HPV infection. The picture was usually diagnostic. It rested chiefly on identifying the koilocyte-the cell with the perinuclear halo that carries the viral antigen in its nucleus-but abnormal keratinisation was also a feature. The accompanying epithelial findings ranged from normal to CIN III (cervical intraepithelial neoplasia). The latter was of an unusual but distinct appearance, in which cytoplasmic maturation was preserved to some degree but in which gross nuclear atypia was seen in all layers of the epithelium.

The koilocyte was first described by Koss and Durfee in $1956,{ }^{\prime}$ and its association with exophytic and flat wart virus infections of the female genital tract was established on cytology by Meisels and Fortin in $1976^{2}$ and Purola and Savia in $1977,{ }^{3}$ with colposcopic and histological confirmation following in 1977.4 The findings of koilocytosis and certain dyskeratotic changes - for example, keratosis and individual cell keratinisation-are believed by many to be specific for papillomavirus infection of the cervical squamous epithelium. Some of these changes may not be peculiar to human papillomavirus (HPV) infections, however, and may occur in other infections.

The specific identification of papillomavirus antigen in cervical epithelium using immunohistochemical techniques ${ }^{56}$ has allowed us to undertake a more objective descriptive study of the morphological changes that are found in epithelium infected with HPV?

\section{Material and methods}

The material came from 200 consecutive patients referred to a colposcopy clinic in north London

Accepted for publication 25 October 1983 because of abnormal smears. Biopsy specimens were taken from 152 women, and material from 139 of them was studied using a broadly cross reactive serum raised by immunisation of a rabbit with disrupted capsids from virions purified from a pool of plantar warts ${ }^{8}$ and an indirect alkaline phosphatase technique. ${ }^{9}$

Of the 139 cases examined, 28 exhibited positive nuclear staining, shown by deep red coloration within the nuclei of cells confined to the upper third of the epithelium. The immediately adjoining sections from each block were stained with haematoxylin and eosin and examined for histological evidence of human papillomavirus infection and for the degree of cervical intraepithelial neoplasia. Details of the methods and results of the study are given elsewhere. ${ }^{10}$

\section{Results}

Cases were classified according to the degree of cervical intraepithelial neoplasia (CIN), " although the degree of koilocytosis rendered the use of the CIN classification difficult in several cases. Histological diagnoses were as follows: benign (two cases); wart virus infection alone (eight cases); CIN I (two cases); CIN II (five cases); CIN III (11 cases).

The finding of positive staining in two of the 


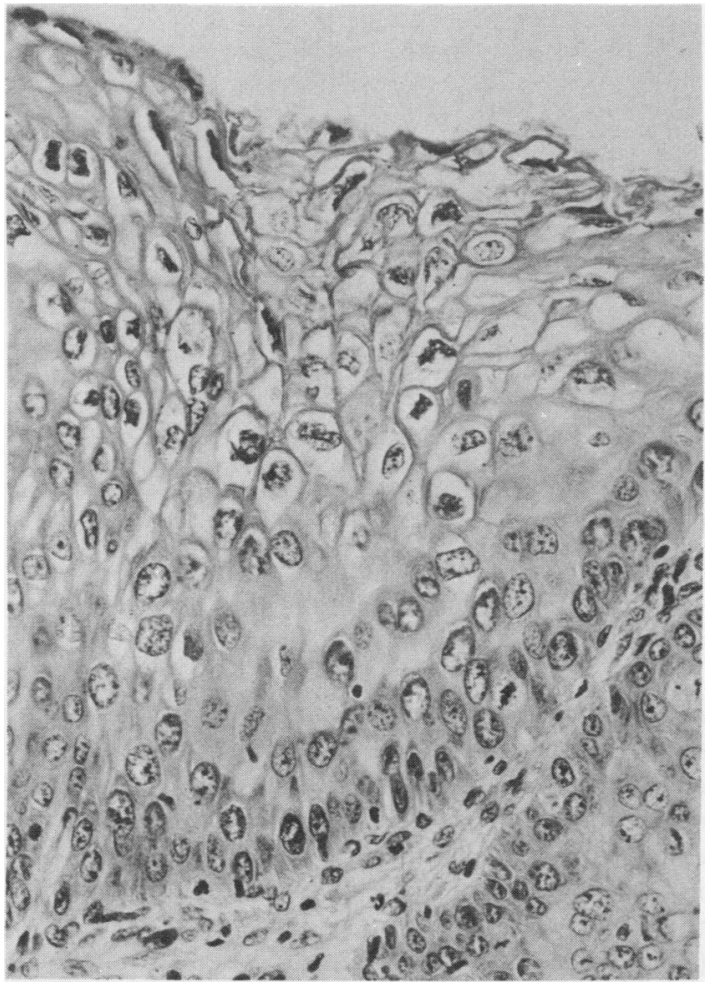

Fig. 1 Koilocytotic epithelium with large perinuclear halo and vesicular nuclei. Haematoxylin and eosin $\times 160$. biopsy specimens classed as benign was surprising. It points to the great difficulty that can arise in distinguishing between HPV infection and mild inflammatory nuclear changes, and it is compounded by the knowledge that squamous epithelia from all sites in the body can show shrinkage of cytoplasm from the nucleus to give an apparent halo.

At the other extreme of dysplasia, the use of the specific stain enabled us to identify koilocytes among the flattened cells of the surface epithelium in CIN III lesions.

Three specific aspects of these biopsy specimens merit consideration in greater detail.

\section{KOILOCYTOSIS}

The nuclei which took the immunohistochemical stain always showed a perinuclear halo. In 26 cases the cells could be readily identified as koilocytes because they showed some degree of nuclear abnormality in association with a perinuclear halo. In two cases virus was expressed in the nuclei of the flattened cells of the surface epithelium, which retained only a small perinuclear halo.

Four degrees of koilocytosis are illustrated: (1) In extreme degrees of koilocytosis (Fig. 1) the perinuclear halo was so huge that it extended to the edge of the cell and even disrupted the cell boundaries. The nucleus was enlarged and vesicular with some chromatin clumping adjacent to the cell membrane. This degree of koilocytosis was characterised by strongly eosinophillic staining and a relatively poor affinity for haematoxylin. Immunohistochemical techniques showed that HPV was being expressed in large amounts by nuclei in the upper third of the epithelium. This is an appearance more

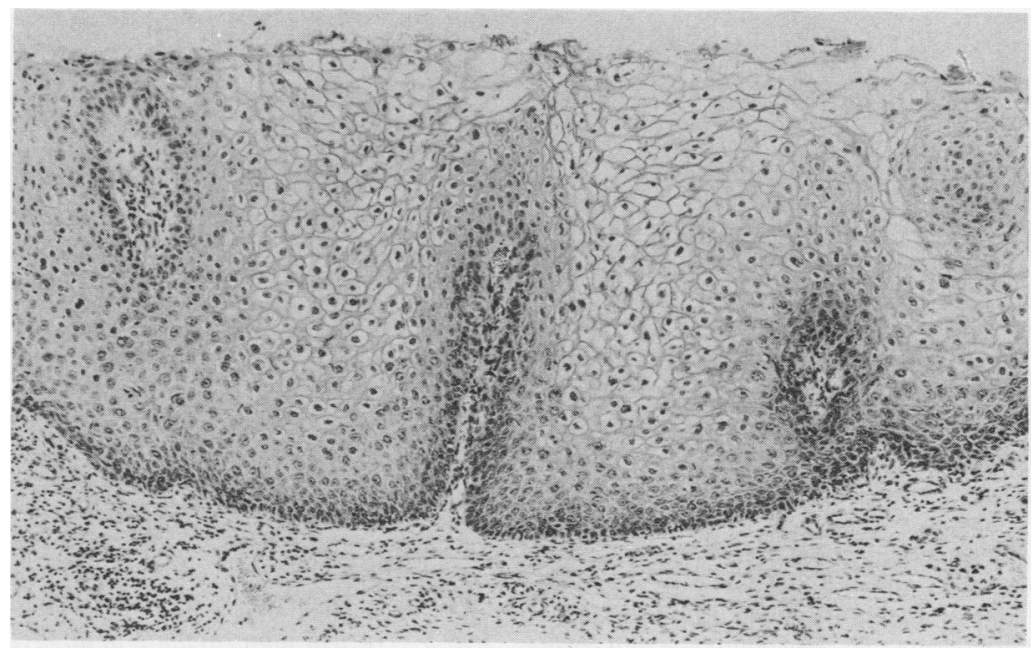

Fig. 2 Flat wart with pronounced koilocytosis. Haematoxylin and eosin $\times 80$. 
commonly seen in exophytic warts, but it is not unknown in flat wart epithelium.

(2) The more usual picture of flat wart epithelium is shown in Fig. 2. The epithelium was acanthotic with prominent dermal papillae. Almost the entire thickness of the epithelium showed koilocytosis, but immunohistochemistry showed the virus only in the superficial layers of the epithelium.

(3) In many cases the epithelium was not thickened. There was sometimes appreciable dysplasia, but polarity and maturation were not completely lost and koilocytes were still identifiable. Fig. 3 shows the following features: the nuclei are large, there is a considerable change in the nuclear-cytoplasmic ratio, and the perinuclear halo is thinned; mitoses are numerous and may be seen halfway up the epithelium; binucleate cells, a feature of HPV infection, are common.

(4) The smallest degree of koilocytes was seen in epithelium which did not show a sufficient degree of abnormality to qualify for a CIN grading. Koilocytes were scanty. Much of the epithelium in the superficial layers was glycogenised, and there were only a few cells which were koilocytotic. The principal abnormality in this biopsy specimen was the presence of prominent cell borders accompanied by intercellular bridges (Fig. 4). This is not a specific feature of HPV infected epithelium, but it may be a valuable aid in distinguising between koilocytotic and glycogenised epithelium. In the latter the cell borders tend to be smudged and indistinct.

DYSKERATOSIS

(1) Parakeratosis is a feature more easily recog-

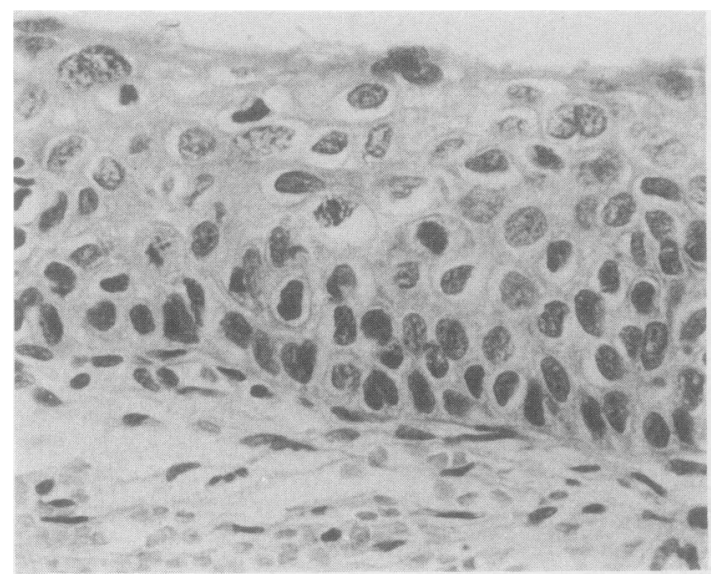

Fig. 3 Relatively thin epithelium showing pronounced nuclear atypia and binucleate cells. Haematoxylin and eosin $\times 400$.

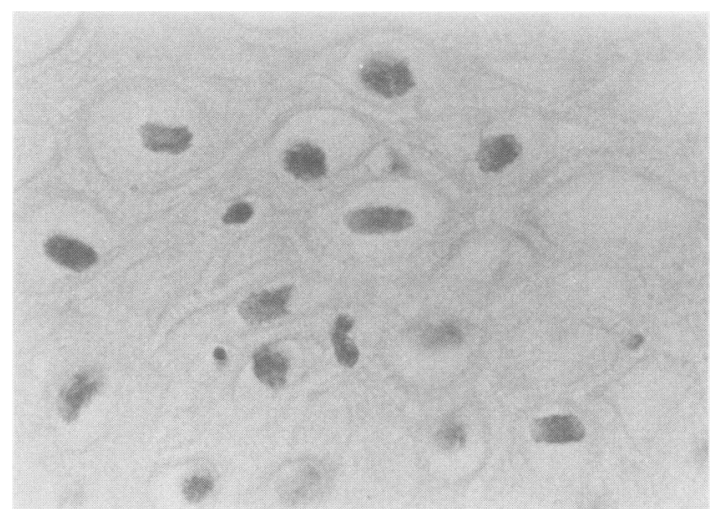

Fig. 4 Prominent intercellular bridges in HPV infected epithelium. Haematoxylin and eosin $\times 1000$.

nised in cytological preparations than in histological slides, and it was first described in connection with cytology of wart virus infections. ${ }^{23}$ Like the development of intercellular bridges, it is another example of the tendency for HPV infection to induce metaplasia to a keratinising type of epithelium.

(2) Individual cell keratinisation is a common feature of flat wart lesions. In this study it was seen in 10 of the 28 cases and was present in all layers of the epithelium.

DYSPLASIA

The 28 cases showed a wide range of dysplasia, rang-

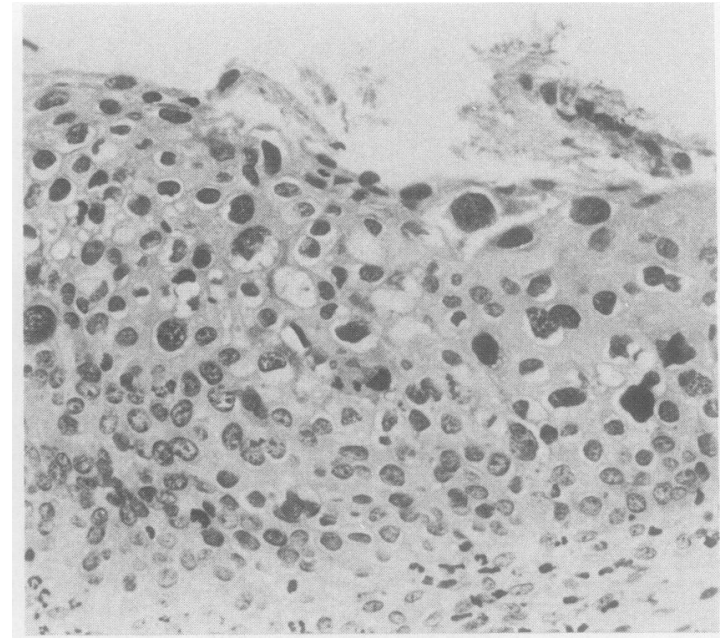

Fig. 5 Nuclear abnormalities in all layers of koilocytotic epithelium. Haematoxylin and eosin $\times 250$. 


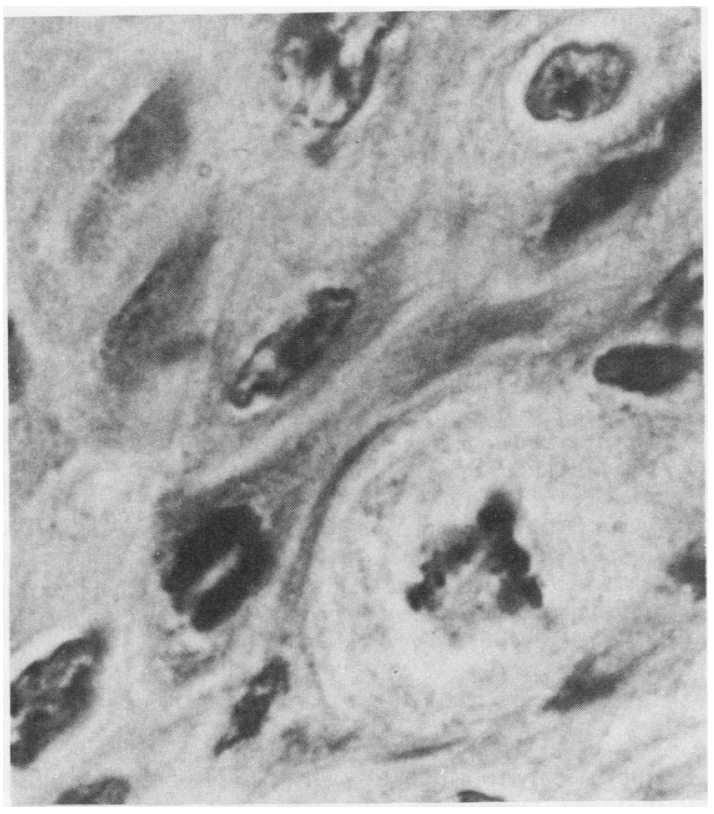

Fig. 6 Atypical mitosis. Haematoxylin and eosin $\times 1000$.

ing from an almost completely normal epithelium to the CIN III shown in Fig. 5. Although retaining a semblance of maturation towards the surface, the epithelium in Fig. 5 is very abnormal. Nuclei were large and hyperchromatic, and mitoses were numerous in all layers of the epithelium. The mitoses were often abnormal, taking tripolar and other nondiploid forms. The cells undergoing mitosis were large and often, as in Fig. 6, had a strikingly pale, glassy cytoplasm. There was a discordance between the architectural arrangement of the epithelium, which retained some polarity, and the nuclear changes, which suggested a more frankly malignant picture.

\section{Discussion}

This paper illustrates the great variety of histological appearances that can be seen in cervical squamous epithelium infected with HPV. It emphasises the crucial importance of the koilocyte (the cell which expresses the virus) in making the diagnosis. The morphology of the koilocyte in tissue sections has been discussed before.$^{12} 13$ The use of a specific antiserum has enabled us to enlarge the range of cells acceptable as koilocytes. The very least abnormality, such as is seen in Fig. 4, may be the only indication of HPV infection. It is the picture commonly seen in colposcopic biopsy specimens of the apparently normal cervixes of women who have vulval warts.
The recognition of koilocytes in the extreme superficial layers of CIN III lesions (Fig. 5), in cases where the large nucleus and flattening of the cell make recognition of koilocytosis difficult, is also of value. To recognise this as a picture associated with wart virus infection may be to delineate a group of women whose CIN III lesions may behave in a different manner from other CIN III lesions, although only follow up over years will clarify their natural progression.

This study has also shown the tendency of epithelium infected by HPV to undergo metaplasia to a keratinising form. Keratinisation appears in three principal ways: the formation of intercellular bridges, parakeratosis, and individual cell keratinisation. This may have a bearing on the natural history of the disease since invasive carcinoma is more likely to arise from a keratinising CIN III than from non-keratinised epithelium.

A third feature to emerge is the degree of nuclear atypicality which may be associated with HPV infection. This is seen in the large hyperchromatic nuclei, the altered nuclear-cytoplasmic ratio, and the frequent atypical mitoses shown in Fig. 3, 6, and 7. This form of CIN III is recognised as a specific variant and is described, in a review of nomenclature of cervical intraepithelial neoplasia," as Burghardt's "large cell undifferentiated keratinising type," in which cytoplasmic maturation is preserved but nuclear abnormalities are present in all layers. More recently, ${ }^{14}$ the non-diploid mitoses have been recognised as a specific feature of HPV 16 infection.

The natural history of these lesions is unclear. A review of human papillomavirus ${ }^{15}$ has shown that in humans, as in animals, some papillomaviruses predispose to malignant change while others regress. The outcome of genital wart virus infections remains guarded. ${ }^{16}$ We hope that by defining the changes associated with HPV infection this study will help towards understanding the problem.

Patrick Walker is supported by the Florence and William Blair Bell Memorial Research Fellowship of the Royal College of Obstetricians and Gynaecologists. This work was supported in part by NIH Grant 1 POI AI 16959 and by a grant from the Cancer Research Campaign (UK) to St Mary's Hospital Medical School (Exp Path 4). We thank Professor Keerti Shah from the Department of Immunology and Infectious Diseases of Johns Hopkins University, Baltimore, USA, who kindly donated the antiserum used in this study. We also thank Dr Dulcie Coleman of the Department of Pathology, St Mary's Hospital, London, for her help and advice and for providing the laboratory facilities for the immunohistochemical staining to be carried out. We 
are grateful to Mr Yabsley, Mrs Perry, and their staff for technical assistance and to Mrs Obee for typing this paper.

\section{References}

' Koss LG, Durfee GR. Unusual patterns of squamous epithelium of the uterine cervix. Cytologic and pathologic studies of koilocytotic atypia. Ann NY Acad Sci 1956;63:1245-61.

${ }^{2}$ Meisels A, Fortin R. Condylomatous lesions of the cervix and vagina. I Cytologic patterns. Acta Cytol 1976;20:505-9.

${ }^{3}$ Purola E, Savia E. Cytology of gynaecologic condyloma acuminatum. Acta Cytol 1977;21:26-31.

${ }^{4}$ Meisels A, Fortin R, Roy M. Condylomatous lesions of the cervix. II Cytologic, colposcopic and histopathologic study. Acta Cytol 1977;21:379-90.

${ }^{5}$ Woodruff JD, Braun L, Cavalieri R, Gupta P, Pass F, Shah KV. Immunologic identification of papillomavirus antigen in condyloma tissues from the female genital tract. Obstet Gynecol 1980;56:727-32.

${ }^{\circ}$ Kurman RJ, Shah KH, Lancaster WD, Jenson AB. Immunoperoxidase localisation of papillomavirus antigens in cervical dysplasia and vulvar condylomas. Am J Obstet Gynecol 1981;140:931-5.

' Walker PG, Singer A, Dyson JL, Shah KV, To A, Coleman DV. The prevalence of human papillomavirus antigen in patients with cervical intraepithelial neoplasia. $B r J$ Cancer 1983;48:99-101.

${ }^{8}$ Jenson AB, Rosenthal JD, Olson C, Pass F, Lancaster WD, Shah $\mathrm{KV}$. Immunological relatedness of papillomaviruses from different species. JNCI 1980;64:495-500.
9 To A, Dearnley DP, Ormerod MG, Kanti G, Coleman DV Indirect-alkaline phosphatase staining of cytological smears of serous effusions for tumour marker studies. Acta Cytol 1983;27:109-17.

${ }^{10}$ Walker PG, Singer A, Dyson JL, Shah KV, Wilters J, Coleman DV. Colposcopy in the diagnosis of papillomavirus infection of the uterine cervix. Br J Obstet Gynaecol 1983;90:1082-6.

"Buckley CH, Butler EB, Fox H. Cervical intraepithelial neoplasia. J Clin Pathol 1982;35:1-13.

12 Reid R, Stanhope CR, Herschman BR, Booth E, Phibbs GD, Smith JP. Genital warts and cervical cancer. 1 Evidence of an association between subclinical papillomavirus infection and cervical malignancy. Cancer 1982;50:377-87.

${ }^{13}$ Fletcher S. Histopathology of papillomavirus infection of the cervix uteri: the history, taxonomy, nomenclature and reporting of koilocytotic dysplasias. J Clin Pathol 1983;36:616-24.

${ }_{14}$ Zoler ML. Human papillomavirus linked to cervical (and other) cancers. JAMA 1983;249:2997-9.

${ }^{15}$ Howley PM. The human papillomaviruses. Arch Pathol Lab Med 1982;106:429-32.

${ }^{16}$ Meisels A, Morin C, Casas-Cordero M. Human papillomavirus infection of the uterine cervix. International Journal of Gynecological Pathology 1982;1:75-94.

Requests for reprints to: $\mathrm{Dr}$ JL Dyson, Consultant Pathologist, Department of Histopathology and Cytology, Whittington Hospital, St Mary's Wing, Highgate Hill, London N19 5NF, England. 